\title{
Adaptive evolution and metabolic engineering of a cellobiose- and xylose- negative Corynebacterium glutamicum that co-utilizes cellobiose and xylose
}

\section{Open Acces:}

Jungseok Lee', Jack N. Saddler ${ }^{2}$, Youngsoon Um ${ }^{1,3}$ and Han Min Woo $13,34^{*}$

\begin{abstract}
Background: An efficient microbial cell factory requires a microorganism that can utilize a broad range of substrates to economically produce value-added chemicals and fuels. The industrially important bacterium Corynebacterium glutamicum has been studied to broaden substrate utilizations for lignocellulose-derived sugars. However, C. glutamicum ATCC 13032 is incapable of PTS-dependent utilization of cellobiose because it has missing genes annotated to $\beta$-glucosidases (bG) and cellobiose-specific PTS permease.

Results: We have engineered and evolved a cellobiose-negative and xylose-negative C. glutamicum that utilizes cellobiose as sole carbon and co-ferments cellobiose and xylose. NGS-genomic and DNA microarray-transcriptomic analysis revealed the multiple genetic mutations for the evolved cellobiose-utilizing strains. As a result, a consortium of mutated transporters and metabolic and auxiliary proteins was responsible for the efficient cellobiose uptake. Evolved and engineered strains expressing an intracellular bG showed a better rate of growth rate on cellobiose as sole carbon source than did other bG-secreting or bG-displaying C. glutamicum strains under aerobic culture. Our strain was also capable of co-fermenting cellobiose and xylose without a biphasic growth, although additional pentose transporter expression did not enhance the xylose uptake rate. We subsequently assessed the strains for simultaneous saccharification and fermentation of cellulosic substrates derived from Canadian Ponderosa Pine.
\end{abstract}

Conclusions: The combinatorial strategies of metabolic engineering and adaptive evolution enabled to construct $C$. glutamicum strains that were able to co-ferment cellobiose and xylose. This work could be useful in development of recombinant C. glutamicum strains for efficient lignocellulosic-biomass conversion to produce value-added chemicals and fuels.

Keywords: Corynebacterium glutamicum, Cellobiose and xylose, Cofermentation, Intracellular $\beta$-glucosidase, Adaptive evolution

\section{Background}

Advances in metabolic engineering and synthetic biology have opened-up opportunities for us to engineer microbial hosts to produce a range of industrially-relevant chemicals and fuels [1, 2]. In addition, oligonucleotide-mediated

\footnotetext{
*Correspondence: hmwoo@kist.re.kr

${ }^{1}$ Clean Energy Research Center, Korea Institute of Science

and Technology (KIST), Hwarangro 14-gil 5, Seongbuk-gu, Seoul 02792 ,

Republic of Korea

Full list of author information is available at the end of the article
}

or CRISPR-CAS9-mediated genome editing technologies have been used to accelerated genomic evolution and enhance development of new strains [3, 4]. More efficient utilization of hexose and pentose derived sugars from lignocellulosic biomass (cellulose: $\sim 48 \%$, xylan: $\sim 22 \%$, lignin: $25 \%$ ) [5] is advantageous to achieve economically-attractive bioprocesse for improving titers, productivities, and yields of value-added chemicals.

An industrial amino acid producer, Corynebacterium glutamicum [6] showed a broad range of sugar utilization 
such as hexose (i.g. glucose and gluconate) and disaccharide (i.g. maltose and sucrose) but some pentose (arabinose but xylose) [7]. Since there are great potentials of $C$. glutamicum as a microbial cell factory to produce other commercially relevant chemicals and fuels [8-10] from renewable lignocellulosic biomass, efficient utilization of cellulosic sugars is an inevitable goal. C. glutamicum has been successfully engineered for cell growth and production of biochemical using pentose sugars via either the heterologous xylose-isomerase pathway $[11,12]$ or Weimberg pathway [13]. For cellobiose utilization in $C$. glutamicum, recent genome sequencing of C. glutamicum R strain showed possible gene clusters that encode for functional EII permeases of PTS (BglF1 and BglF2), and for functional phospho- $\beta$-glucosidases (BglG1 and BglG2) [14]. Thus, an adaptive strain of C. glutamicum $\mathrm{R}$ strain grown in minimal medium with $0.2 \%$ cellobiose and glucose has revealed a single-substitution mutation $\operatorname{BglF}(\mathrm{V} 217 \mathrm{~A}$ or $\mathrm{V} 317 \mathrm{M})$ for cellobiose utilization [14, 15]. The C. glutamicum R-CEL strain has been shown to utilize cellobiose, glucose and xylose simultaneously, but only possible under anaerobic conditions [16].

However, C. glutamicum ATCC 13032 is incapable of PTS-dependent utilization of methyl $\beta$-D-glucoside and cellobiose because it does not have any genes annotated to $\beta$-glucosidases (bG). In addition, no genes encoding for cellobiose-specific PTS permease were annotated [17]. To metabolize cellobiose, C. glutamicum ATCC 13032 must have an enzyme that cleaves the $\beta$ - $(1 \rightarrow 4)$-glycosidic linkage of cellobiose. Thus, bG-displaying or secreting C. glutamicum strains have been developed and exhibited the complete consumption of $20 \mathrm{~g} / \mathrm{L}$ cellobiose in 4 days with L-lysine production [18]. The cellobiose utilization was quite slow, compared to the glucose consumption of current C. glutamicum strains. Thus, optimizing gene expression and maximizing the activity of bG was necessary for better production of L-lysine and other chemicals. Recently, Saccharomyces cerevisiae (a cellobiose- and xylose-negative yeast strain) was engineered for cellobiose utilization by expressing cellodextrin transporter (CDT-1) and intracellular bG along with a xylose-consuming pathway in order to resolve carbon catabolite repression by glucose in hydrolysates [19]. This engineering has enabled simultaneous utilization of cellobiose and xylose as a model hydrolysate, and increased the productivity of ethanol.

Like S. cerevisiae, C. glutamicum ATCC 13032 is not able to utilize either cellobiose or xylose as sole carbon source. First, we performed metabolic engineering of C. glutamicum for cellobiose utilization by expressing a cellodextrin transporter and an intracellular bG (Fig. 1) and evolved the strains for the efficient cellobiose utilization. Subsequently, NGS-genomic and DNA microarray transcriptomic analysis were performed to characterize the evolved strains. Next, we have introduced the xyloseisomerase pathway $[11,12,20]$ to the cellobiose-utilizing C. glutamicum strain for co-utilization of cellobiose and xylose. Our cellobiose-utilizing engineered strains were used to ferment the cellobiose and glucose derived from Canadian Ponderosa Pine in simultaneous saccharification and fermentation (SSF). Canadian Ponderosa Pine was used as a model lignocellulosic biomass.

\section{Results and discussion}

Utilization of cellobiose in C. glutamicum using metabolic engineering and adaptive evolution

To test whether heterologous expressions of either CDT-1 transporter and bG or bG alone allow utilization of cellobiose in C. glutamicum, the N. crassa cdt-1 and gh1-1 gene were codon-optimized and introduced into a CoryneBrick vector [11], pBbEB1c (Table 1). We attempted to grow $\mathrm{Cg}$-CelloO1 strain containing both the $c d t-1$ and $g h 1-1$ genes, and $\mathrm{Cg}$-CelloO2 containing the gh1-1 gene in CgXII minimal medium containing $2 \%$ $(\mathrm{w} / \mathrm{v})$ cellobiose as sole carbon source. No growth of $\mathrm{Cg}$ Cello01 was observed for $4 \mathrm{~d}$. Surprisingly, the cultures of $\mathrm{Cg}$-CelloO1 strain exhibited the growth only after day 16 (Fig. 2). As soon as we observed the maximal cell growth of each strain (corresponding to the growth in presence of $2 \%$ glucose), the adapted strain was transferred to fresh CgXII minimal medium containing $2 \%$ $(\mathrm{w} / \mathrm{v})$ cellobiose for $48 \mathrm{~h}$. After the first transfer, the growth and residual sugars in the culture medium were determined for each cell culture. However, no cell growth was observed for $C g-p B b E B 1 c$ as a control. During the adaptive evolution of $\mathrm{Cg}$-CelloO1 strain by three serial transfers, cellobiose was gradually consumed during cell cultures (Fig. 2b). Interestingly, glucose in the medium was detected up to $12 \mathrm{~g} / \mathrm{L}$ during the evolution and then glucose derived from cellobiose was consumed after cellobiose was completely depleted. In the last evolutionary round (third serial transfer), Cg-Cello01(evo) showed the complete cellobiose consumption in $12 \mathrm{~h}$ and glucose $(5 \mathrm{~g} / \mathrm{L})$ was minimally secreted.

On the other hand, fewer adaptations of $\mathrm{Cg}$-CelloO2 were performed to obtain $\mathrm{Cg}$-CelloO2(evo). $\mathrm{Cg}$-CelloO2 strains exhibited the growth after day 11 . Only twice serial transfers were performed to obtain the desired phenotype, of which the strain completely consumed its cellobiose in $12 \mathrm{~h}$. No glucose derived from cellobiose was secreted during the adaptive evolution rounds (Fig. 2c). Finally, we confirmed no phenotypic changes (cell growth and cellobiose consumption) shown over more than twenty's serial transfers into fresh medium (not shown) after $\mathrm{Cg}$-Cello01(evo) and $\mathrm{Cg}$-Cello02(evo) were obtained. As a result, the patterns of cell growth and 


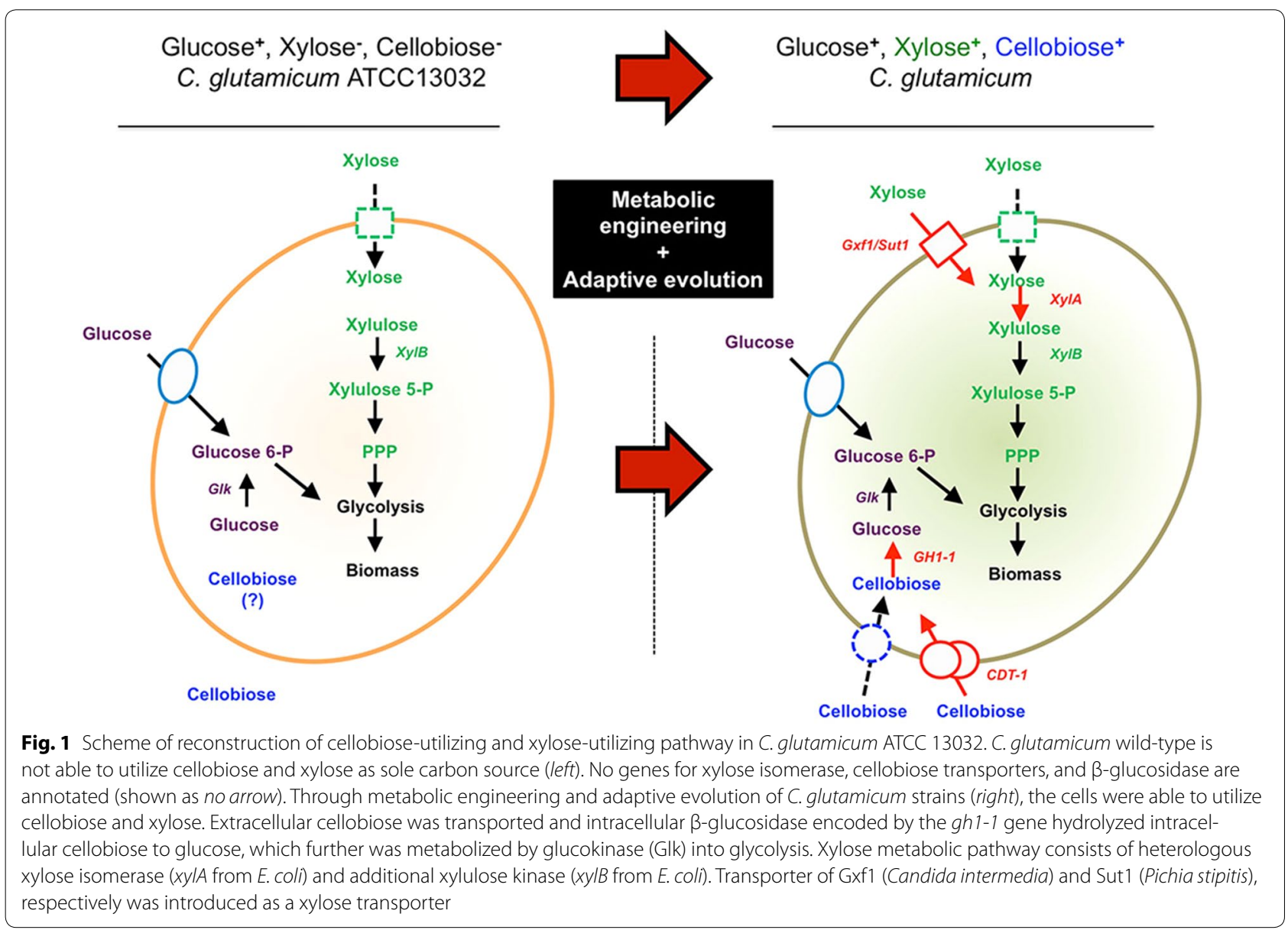

$2 \%$ (w/v) sugar consumption of Cg-Cello01(evo) and $\mathrm{Cg}$ Cello01(evo) were almost identical to those of the wildtype [11], regardless of cellobiose or glucose. Finally, we obtained $\mathrm{Cg}$-CelloO1(evo) and Cg-CelloO2(evo) strains as cellobiose-utilizing C. glutamicum ATCC 13032 derivative strains. $\mathrm{Cg}$-CelloO1(evo) strain is the fastest cellobiose-utilizing strain known under aerobic conditions.

Cellobiose-utilizing C. glutamicum requires activities of bG and glucokinase (Glk) for the catabolism of cellobiose in the cytosol. Thus, we checked whether the bG and Glk activities of the Cg-Cello01(evo) and Cg-Cello02(evo) strains were altered (Fig. 3). As a result, the control $(C g-p B b E B 1 c$; the wild-type carrying the empty vector) showed no bG activity (both cell extract and supernatant). On the other hand, levels of the bG activities in the cell extracts of $\mathrm{Cg}$-CelloO1(evo) and $\mathrm{Cg}$-CelloO2(evo) grown on $2 \%$ cellobiose were measured at $0.2 \pm 0.01$ and $0.17 \pm 0.002 \mathrm{U} / \mathrm{mg}$, respectively (Fig. 3). Significantly low or none of the bG activities were seen in the cell-free supernatants from $\mathrm{Cg}$-Cello01(evo) or Cg-CelloO2(evo) cultures. Also, the bG activity levels were quite similar to a bG activity from wild-type expressing GH-1-1 alone (initial $\mathrm{Cg}$-CelloO2) was grown on $2 \%$ glucose, the bG activity $(0.17 \pm 0.01)$. Thus, these results indicated that the adaptive evolution did not alter the intracellular expression of heterologous bG. Also, no exogenous expression of bG was occurred due to possible genetic mutations. In addition, we measured Glk activity over the culture interval until the carbon sources were depleted. The Glk activity of $\mathrm{Cg}$-CelloO1(evo) and $\mathrm{Cg}$-CelloO2(evo) was not significantly different the Glk activity from $\mathrm{Cg}$ $p B b E B 1 c$. Therefore, having bG activity in the cytosol of C. glutamicum is one of key steps to utilization of cellobiose, but increasing or high bG activity is not necessary for better cellobiose utilization.

\section{Characterization of the evolved cellobiose-positive C. glutamicum strains}

Through metabolic engineering and adaptive evolution of C. glutamicum, we obtained the cellobiose-utilizing strains, $\mathrm{Cg}$-Cello01(evo) and $\mathrm{Cg}$-CelloO2(evo). Since $\mathrm{C}$. glutamicum wild-type does not have any genes annotated 
Table 1 Bacteria strains and plasmids used in this study

\begin{tabular}{|c|c|c|}
\hline Strain or plasmid & Relevant characteristics & Source or reference \\
\hline \multicolumn{3}{|l|}{ Strains } \\
\hline E. coli HIT-DH5a & $\mathrm{F}^{-}\left(80 \mathrm{~d}\right.$ lacZ M15) (lacZYA-argF) U169 hsdR17 $\left(\mathrm{r}^{-} \mathrm{m}^{+}\right)$recA1 endA1 relA1 deoR96 & [38] \\
\hline C. glutamicum ATCC 13032 & C. glutamicum wild-type strain (ATCC 13032) & ATCC \\
\hline$C g-p B b E B 1 c$ & C. glutamicum wild-type harboring pBbEB1 c, $\mathrm{Cm}^{r}$ CoryneBrick empty vector & [39] \\
\hline$C g-E C X y \mid A B$ & C. glutamicum wild-type harboring pBbEB1c-EcXylA(cg.co) $-E c X y \mid B(c g . c o), \mathrm{Cm}^{r}$ & [11] \\
\hline Cg-Cello01 & C. glutamicum wild-type harboring pBbEB1c-CT-bG, $\mathrm{Cm}^{r}$ & This study \\
\hline Cg-Cello02 & C. glutamicum wild-type harboring pBbEB1 c-bG, $\mathrm{Cm}^{r}$ & This study \\
\hline Cg-Cello01(evo) & Cellobiose-adapted Cg-Cello01 strain, $\mathrm{Cm}^{r}$ & This study \\
\hline Cg-Cello02(evo) & Cellobiose-adapted Cg-Cello02 strain, $\mathrm{Cm}^{r}$ & This study \\
\hline Cg-Evol & Cellobiose-adapted Cg-Cello01 strain after plasmid curing (plasmid-free), cellobiose ${ }^{-}, \mathrm{Cm}^{\mathrm{s}}$ & This study \\
\hline Cg-Evo2 & Cellobiose-adapted $\mathrm{Cg}$-Cello02 strain after plasmid curing (no plasmids), cellobiose ${ }^{-}, \mathrm{Cm}^{\mathrm{s}}$ & This study \\
\hline Cg-Cello03 & Cg-Evol harboring pBbEB1c-bG, $\mathrm{Cm}^{r}$ & This study \\
\hline Cg-Cello04 & Cg-Evo2 harboring pBbEB1c-bG, $\mathrm{Cm}^{r}$ & This study \\
\hline Cg-Cello03-Xyl01 & Cg-Cello03 harboring pBbEB1c-bG-XIK, $\mathrm{Cm}^{r}$ & This study \\
\hline Cg-Cello03-Xyl02 & Cg-Cello03 harboring pBbEB1c-bG-XIK-XTg, $\mathrm{Cm}^{r}$ & This study \\
\hline Cg-Cello03-Xyl03 & Cg-Cello03 harboring pBbEB1c-bG-XIK-XTs, $\mathrm{Cm}^{r}$ & This study \\
\hline Cg-Cello04-Xyl01 & Cg-Cello04 harboring pBbEB1c-bG-XIK, $\mathrm{Cm}^{r}$ & This study \\
\hline Cg-Cello04-Xyl02 & Cg-Cello04 harboring pBbEB1c-bG-XIK-XTg, $\mathrm{Cm}^{r}$ & This study \\
\hline Cg-Cello04-Xyl03 & Cg-Cello04 harboring pBbEB1c-bG-XIK-XTs, $\mathrm{Cm}^{r}$ & This study \\
\hline \multicolumn{3}{|l|}{ Plasmids } \\
\hline pBbEB1c & ColE1 (Ec), pBL1 (Cg), Cmr, P trc $_{1}$ BglBrick sites, CoryneBrick vector & [39] \\
\hline pBbEB1c-CT-bG & pBbEB1c derivative containing each codon-optimized N. crassa cdt1 and gh 1-1 genes & This study \\
\hline pBbEB1c-bG & pBbEB1c derivative containing each codon-optimized N. crassa gh 1-1 gene & This study \\
\hline pBbEB1c-bG-XIK & $\begin{array}{l}\text { pBbEB1c derivative containing each codon-optimized N. crassa gh 1-1 gene and E. coli xylA and } \\
\text { xy/B genes }\end{array}$ & This study \\
\hline pBbEB1c-bG-XIK-XTg & $\begin{array}{l}\text { pBbEB1c derivative containing each codon-optimized N. crassa gh 1-1 gene and E. coli xylA and } \\
\text { xy/B genes and C. intermedia gxf1 gene }\end{array}$ & This study \\
\hline pBbEB1c-bG-XIK-XTs & $\begin{array}{l}\text { pBbEB1c derivative containing each codon-optimized N. crassa gh } 1-1 \text { gene and E. coli xylA and } \\
x y / B \text { genes and P. stipitis sut1 gene }\end{array}$ & This study \\
\hline
\end{tabular}

cellobiose transporter, we investigated if the evolved cells have either functional CDT-1 transporters or altered transporters for the uptake of cellobiose.

First, plasmids used for metabolic engineering were isolated to characterize genetic mutations occurred during the adaptive evolution. When we compared the original sequence of plasmids, pBbEB1c-CT-bG and pBbEB1c-bG, in-frame deletion and a point mutation were found in the region of replication of origins (Additional file 1: Figure S1), but no mutations were found on the sequence of the gh1-1 gene. Interestingly, the $c d t$ 1 gene that was present on the pBbEB1c-CT-bG in the Cg-Cello01 strain was missing, which was confirmed by DNA sequencing. This could be due to intra-molecular recombination that occurs during the adaptive evolution at the identical and ribosomal binding synthetic DNA sequences of the $c d t-1$ and $g h 1-1$ genes. Also, the gel images of the $c d t-1$ gene by colony PCR were shown for the loss of $c d t-1$ gene during the evolutionary process of the Cg-CelloO1 to Cg-Cello01(evo) strains (Additional file 1: Figure S2). This result indicated that heterologous expression of CDT-1 was not suitable for the cellobiose uptake in C. glutamicum. Also, we found that there were no mutations found in the sequence of the gh1-1 gene encoding for bG on the plasmids although a substitution mutation of BglF (V217A or V317 M) was found on the adaptive $C$. glutamicum $\mathrm{R}$ strain [15]. Thus, the plasmid sequencing results confirmed that the intracellular expression of heterologous bG was sufficient to utilize cellobiose as sole carbon source in Cg-CelloO1(evo) and Cg-Cello02(evo) strains (Fig. 3).

To characterize the genetic basis of cellobiose-utilizing C. glutamicum, next-generation sequencing (NGS) analysis was applied to fully-evolved $\mathrm{Cg}$-Cello01(evo) and $\mathrm{Cg}$-CelloO2(evo) strains, compared with the reference genome sequence of C. glutamicum ATCC 13032 (Additional file 1: Table S1 and Table S2). As a result, in the genome sequence of $\mathrm{Cg}$-CelloO1(evo) strain thirtysix genes were mutated with thirty-one single-nucleotide variants including missense (15 variants) and 

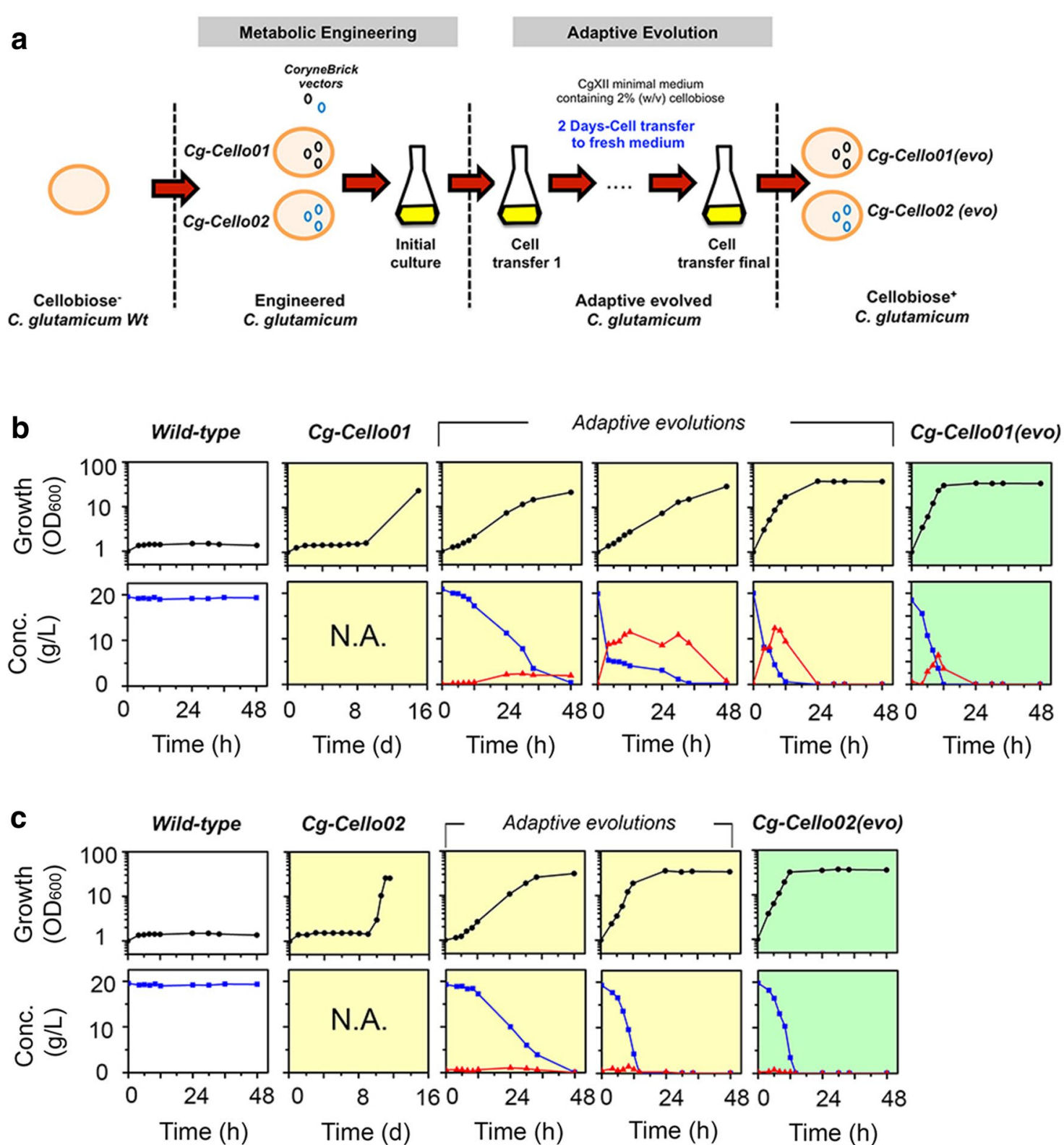

Fig. 2 Adaptive evolution of engineered C. glutamicum strains. a Scheme of metabolic engineering and adaptive evolution of $\mathrm{Cg}$-Cello01 and $\mathrm{Cg}$-Cello02 strains were described. CoryneBrick vectors containing the $\mathrm{cdt}$-1 and/or gh1-1 gene(s) were introduced into C. glutamicum wild-type, in which no growth and consumption of cellobiose were observed (b and $\mathbf{c}$; the first column). Growth of Cg-Cello01 and Cg-Cello02 strain was not appeared initially. However, the maximal cell growths of $\mathrm{Cg}$-Cello01 and $\mathrm{Cg}$-Cello02 were observed after $16 \mathrm{~d}$ ( $\mathbf{b}$; the second column) and after $11 \mathrm{~d}(\mathbf{c}$; the second column), respectively. Subsequently, serial cell transfers were performed for adaptive evolutions of $\mathrm{Cg}$-Cello01 and $\mathrm{Cg}$-Cello02 in $48 \mathrm{~h}$ (b and c). Finally, Cg-Cello01 (evo) and Cg-Cello02(evo) were obtained since growth and cellobiose consumption were unchanged. Growth at $\mathrm{OD}_{600}$, $\mathrm{Cel}^{-}$ lobiose $(\mathrm{g} / \mathrm{L})$ and glucose $(\mathrm{g} / \mathrm{L})$ were shown in a symbol of circle (black), square (blue) and triangle (red), respectively. Data represents mean values of at least three cultivations. (N.A.) not available

silent mutations (five variants) in the coding regions, two multi-nucleotide variants, one insert and two deletions (Additional file 1: Table S1). Cg-Cello02(evo) strain exhibiting shorter adaption were shown for more mutations occurred. Three hundred single-nucleotide variants including missense (123 variants), nonsense (six variants), silent mutations (98 variants) in the coding regions were identified along with 41 insertions and 28 deletions in the nucleotide sequence. Yet, the reasons for the high number of mutations for the evolved strains were unclear 

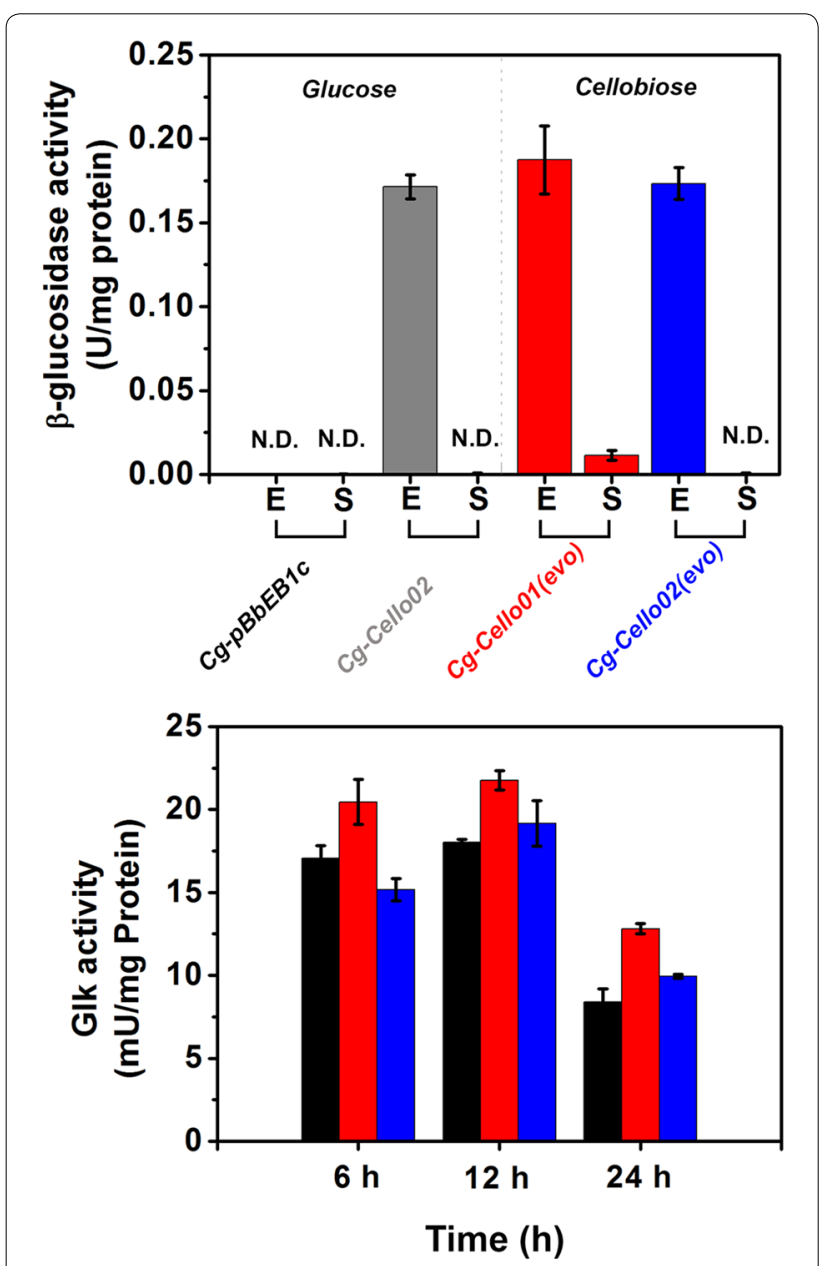

Fig. 3 Measurement of $\beta$-glucosidase (bG) and glucokinase (Glk) activities in C. glutamicum wild-type or evolved strains. The $\mathrm{Cg}$-pBbEB1c (black) and Cg-Cello02 (grey) were cultivated in CgXII medium with $2 \%(\mathrm{w} / \mathrm{V})$ glucose. The evolved Cg-Cello01 (evo) (red) and $\mathrm{Cg}$-Cello02(evo) (blue) strains were cultivated in CgXII medium with $2 \%(\mathrm{w} / \mathrm{v})$ cellobiose. The cell extract (E) and cell-free supernatant $(\mathrm{S})$ were used for the measurement of the bG activities $(\mathrm{U} / \mathrm{mg}$ protein) when the strains were cultivated for $24 \mathrm{~h}$ (upper panel). The cell extracts from the strains grown for 6,12 , or $24 \mathrm{~h}$ were used for the measurement of the Glk activities ( $\mathrm{mU} / \mathrm{mg}$ protein) (lower panel) Data represents mean values of at least three cultivations and error bars represent standard deviations. (N.D.) not detected

since the complete genome sequences of cellobioseutilizing C. glutamicum R-CEL and CEL2 strains were not available $[14,15]$. Specifically, multiple genetic variants were found in genes encoding for putative proteins, phage integrase, ATPase component of ABC-type transport system (Cg2184), GTPase, tranposase (Cg2461), and intergentic regions. Thus, we searched the identical genetic variants in between $\mathrm{Cg}$-Cello01(evo) and Cg-Cello02(evo) strains. Finally, 10 identical genetic variants were identified (Table 2). Three genes encoding for membrane proteins (ABC-type transporter, RibX, LysEtype transloactor) were shown for the missense mutations, which could be responsible membrane proteins for the cellobiose uptake. In addition, two genes $(w z z$ and fruR) involved in sugar metabolism were mutated, changing its amino acid sequences (Glu363Asp and Gly75Val), respectively. MiaB (tRNA methylthiotransferase (MiaB), Maltose-bninding protein (AmyE), and Benoate 1,2-dioxgenase (BenA) were also confirmed to be mutated. Unlike evolved C. glutamicum R strains, C. glutamicum ATCC1304 required multiple mutations for the cellobiose uptake in a concert of altered membrane proteins, metabolic and regulatory proteins, translational processing, and auxiliary proteins. The mechanism of those muteins for cellobiose utilization remains unclear.

Next, to characterize the genome-wide gene expression of the Cg-CelloO1(evo) and Cg-Cello02(evo) strains utilizing cellobiose, we performed a DNA microarray-transcriptomic analysis to investigate whether the gene-expression levels of sugar transporters, or other membrane proteins, were differentially altered in the evolved strains during cellobiose consumption. We analyzed three groups: (1) gene expression of the evolved strains grown on glucose with a control $(C g-p B b E B 1 c)$ on glucose, (2) gene expression of the evolved strains grown on cellobiose with a control $(C g-p B b E B 1 c)$ on glucose, and (3) gene expression of the evolved strains grown on cellobiose with evolved strains on glucose. Those with gene expression that was 2-fold up- and 0.5-fold downregulated in Group 2 and Group 3 were selected for further analysis (Fig. 4 and Additional file 1: Figure S3 and Table S3). Among 32 and 66 differentially altered genes for Cg-Cello01(evo) and Cg-CelloO2(evo), respectively, the gene expressions of four and fourteen membrane proteins (respectively) were changed significantly. Most of them are hypothetically annotated, according to the National Center for Biotechnology Information (NCBI) (accession no. NC003450).

In a comparison of transcriptomic analysis with nextgeneration sequencing-analysis, the mRNA expression of the regulon of FruR (the $p t s H, p t s F, p f k B 1, p t s I$ gene encoding for EII components of the PTS system) were not altered although the transcriptional FruR has been known to attenuate the induction of EII components of the PTS system in C. glutamicum R [21]. Thus, mutated FurR in the evolved strain may be not functional as a transcriptional regulator. However, the mRNA expression of a DeoR-type transcriptional regulator (FruR, Cg2118) was highly up-regulated (5-fold) in the Cg-CelloO2(evo) strain in presence of cellobiose than glucose (Group 3). Also, the mRNA expression of benoate 1,2-dioxgenase (BenA) was only down-regulated (0.3-fold) in the $\mathrm{Cg}$ Cello02(evo) strain in presence of cellobiose than glucose 
Table 2 List of common mutations of C. glutamicum Cg-Cello01(evo) and Cg-Cello02(evo) strains

\begin{tabular}{|c|c|c|c|c|c|c|c|}
\hline $\begin{array}{l}\text { Reference } \\
\text { position }\end{array}$ & Gene name & Type & $\begin{array}{l}\text { Reference } \\
\text { nucleotide }\end{array}$ & $\begin{array}{l}\text { Allele } \\
\text { nucleotide }\end{array}$ & $\begin{array}{l}\text { Coding region } \\
\text { change }\end{array}$ & $\begin{array}{l}\text { Amino acid } \\
\text { change }\end{array}$ & Annotation \\
\hline 32227 & cg0045 & MISSENSE & A & $\mathrm{T}$ & $551 \mathrm{~A}>\mathrm{T}$ & Asn184lle & $\begin{array}{l}\text { Probable ABC } \\
\text { transport protein, } \\
\text { membrane com- } \\
\text { ponent }\end{array}$ \\
\hline 364912 & cg0414 & MISSENSE & A & $C$ & $1089 A>C$ & Glu363Asp & $\begin{array}{l}\text { Wzz, cell surface } \\
\text { polysaccharide } \\
\text { biosynthesis/chain } \\
\text { length determi- } \\
\text { nant protein }\end{array}$ \\
\hline 1689677 & cg1796 & MISSENSE & C & $\mathrm{T}$ & $103 G>A$ & Glu35Lys & $\begin{array}{l}\text { RibX, putative mem- } \\
\text { brane protein-C. } \\
\text { ammoniagenes } \\
\text { RibX homolog }\end{array}$ \\
\hline 2041951 & $\operatorname{cg} 2118^{\mathrm{a}}$ & MISSENSE & G & $\mathrm{T}$ & $224 G>T$ & Gly75Val & $\begin{array}{l}\text { FruR, transcriptional } \\
\text { regulator of sugar } \\
\text { metabolism, DeoR } \\
\text { family }\end{array}$ \\
\hline 2058943 & cg2135 & MISSENSE & A & G & $490 \mathrm{~T}>\mathrm{C}$ & Ser164Pro & $\begin{array}{l}\text { MiaB, tRNA methyl- } \\
\text { thiotransferase }\end{array}$ \\
\hline 2296630 & cg2380 & Deletion & C & - & 270delG & Gly90 fs & $\begin{array}{l}\text { Hypothetical protein } \\
\text { Cg2380 }\end{array}$ \\
\hline 2331324 & cg2412 & MISSENSE & C & $\mathrm{T}$ & $154 \mathrm{C}>\mathrm{T}$ & Pro52Ser & $\begin{array}{l}\text { Hypothetical protein } \\
\text { Cg2412 }\end{array}$ \\
\hline 2545730 & cg2637b & MISSENSE & G & A & $436 \mathrm{G}>\mathrm{A}$ & Asp146Asn & $\begin{array}{l}\text { BenA, benzoate } \\
\text { 1,2-dioxygenase } \\
\text { alpha subunit } \\
\text { (aromatic ring } \\
\text { hydroxylation } \\
\text { dioxygenase A) }\end{array}$ \\
\hline 2607484 & cg2705 & MISSENSE & G & A & $407 C>T$ & Ala136Val & $\begin{array}{l}\text { AmyE, maltose- } \\
\text { binding protein } \\
\text { precursor }\end{array}$ \\
\hline 2826260 & cg2941 & MISSENSE & $\mathrm{T}$ & C & $577 A>G$ & Ile193Val & $\begin{array}{l}\text { LysE type translo- } \\
\text { cator }\end{array}$ \\
\hline
\end{tabular}

Full list of all mutations of C. glutamicum Cg-Cello01(evo) and Cg-Cello01(evo) strains were described in the Additional file 1: Table S1 and S2

a The mRNA expression of the $c g 2118$ gene was highly up-regulated

b The mRNA expression of the cg2637 gene was highly down-regulated. See the details in the text

(Group 3), in which mRNA expression is repressed by global transcriptional regulator GlxR in sugar metabolism [22].

Since we observed the overexpressed hypothetical membrane proteins from the gene expression profiling of the cellobiose-utilizing strains (Fig. 4), we looked into the fatty acid profiles of cellular membranes of the wildtype strain, $\mathrm{Cg}$-CelloO1(evo), and Cg-CelloO2(evo). Compared to the fatty acid profiles of the wild-type, lower palmitic acids $\left(\mathrm{C}_{16: 0}\right)$ and higher unsaturated steric acids $\left(\mathrm{C}_{18: 1 w 9 \mathrm{c}}\right)$ were measured in the lipids of the cellobioseutilizing strains (Cg-CelloO1(evo) and Cg-CelloO1(evo)) (Table 3). This altered lipid profile of C. glutamicum was also shown for the wild-type growing on sodium oleate, and for mutants [23]. Moreover, cellobiose utilization influenced the fatty acid profiles of a recombinant
Rhodococcus opacus PD630 expressing bG, which accumulated fatty acids from cellobiose [24]. Multiple mutations metabolic and regulatory proteins, translational processing, and auxiliary proteins, intergenic regions in evolved C. glutamicum could be responsible for altered lipid profiles.

Based on the NSG- and transcriptomic analysis, altered ABC-type transporters/hypothetical membrane proteins and sugar metabolism were responsible for efficient cellobiose utilization in C. glutamicum. However, it was difficult to pinpoint which single transporter is mainly designated for the cellobiose uptake. Rather, multiple gene mutations could be required for the efficient cellobiose uptake in C. glutamicum. Those mutated genes in common could be good targets for further engineering of C. glutamicum wild-type to explorer cellobiose uptake 
Fig. 4 Heat map of altered gene expressions of C. glutamicum strains with cellobiose or glucose. Evolved C. glutamicum strains [CgCello01 (evo) and Cg-Cello02(evo)] grown on $2 \%(\mathrm{w} / \mathrm{v})$ cellobiose were tested with either a control (Cg-pBbEB1C) or Cg-Cello01 (evo) and CgCello02(evo) grown $2 \%(\mathrm{w} / \mathrm{v})$ glucose. The mRNA expression changed with 2-fold up- and 0.5-fold down-regulated were selected in the evolved strains with cellobiose over glucose (the third columns) as well as a control (the second columns). The mRNA ratios are averages from at least duplicated experiments. Heat maps generated by MeV (MutiExperiment Viewer ver. 4.8) showed differential gene expression of significantly changed genes. Up-regulated signals relative to the mean were colored in red. Down-regulated were colored in green (scale bar, log 2 of mRNA ratio). The criterion used for selection of RNA ratios was a signal-to-noise ratio of $>3$ for either $C y 5$ fluorescence. For the significantly changed genes, $\mathrm{P}<0.05$ as determined by a one-way ANOVA. The ID numbers of $C$. glutamicum were given at the last column, of which data were described in the Additional file 1: Table S1

and corresponding sugar metabolism or protein structures. Thus, comprehensive next-generation sequencinganalysis could be required to analyze the evolving and evolved strains to investigate the most critical mutations for the cellobiose utilization.

\section{Reconstruction of cellobiose-positive chassis of the adaptive evolved C. glutamicum strains}

Inverse engineering the $C$. glutamicum wild-type is necessary to construct rational microbial cells for cellobiose utilization. However, lack of multiple genome editing technology such as RNA-guided CRISPR-CAS9 [4] or MAGE [3] system of C. glutamicum has led to limitation of inverse engineering of C. glutamicum in this study. Thus, we decided to re-construct a cellobiose-positive chassis in which all multiple genetic changes were already reflected, for further engineering. We obtained plasmidfree strains by curing of plasmids in Cg-Cello01(evo) and $\mathrm{Cg}$-CelloO2(evo), resulting in $\mathrm{Cg}$-Evo1 and $\mathrm{Cg}$-Evo2 (Table 1).

After the cultivation of $C g$-Evo1 and $C g$-Evo2 using $2 \%$ cellobiose as sole carbon source, we confirmed that $\mathrm{Cg}$ Evo1 and $C g$-Evo 2 did not grow at all. Thus, pBbEB1c-bG plasmid was introduced to $C g$-Evo1 and $C g$-Evo2, yielding Cg-CelloO3 and Cg-CelloO4 strains (Fig. 5). Cg-CelloO3 and $\mathrm{Cg}$-CelloO4 strains showed complete growth and consumption of cellobiose in the CgXII medium containing $2 \%(\mathrm{w} / \mathrm{v})$ cellobiose, without any adaptations or preculture with cellobiose (Fig. 5). This result supports that the $C g$-Evo1 and $C g$-Evo2 have already multiple genetic changes from its parental strain for the efficient cellobiose uptake.

When we compared the profiles of growth and cellobiose consumption, the $\mathrm{Cg}$-CelloO3 strain showed almost identical patterns of growth and cellobiose consumption

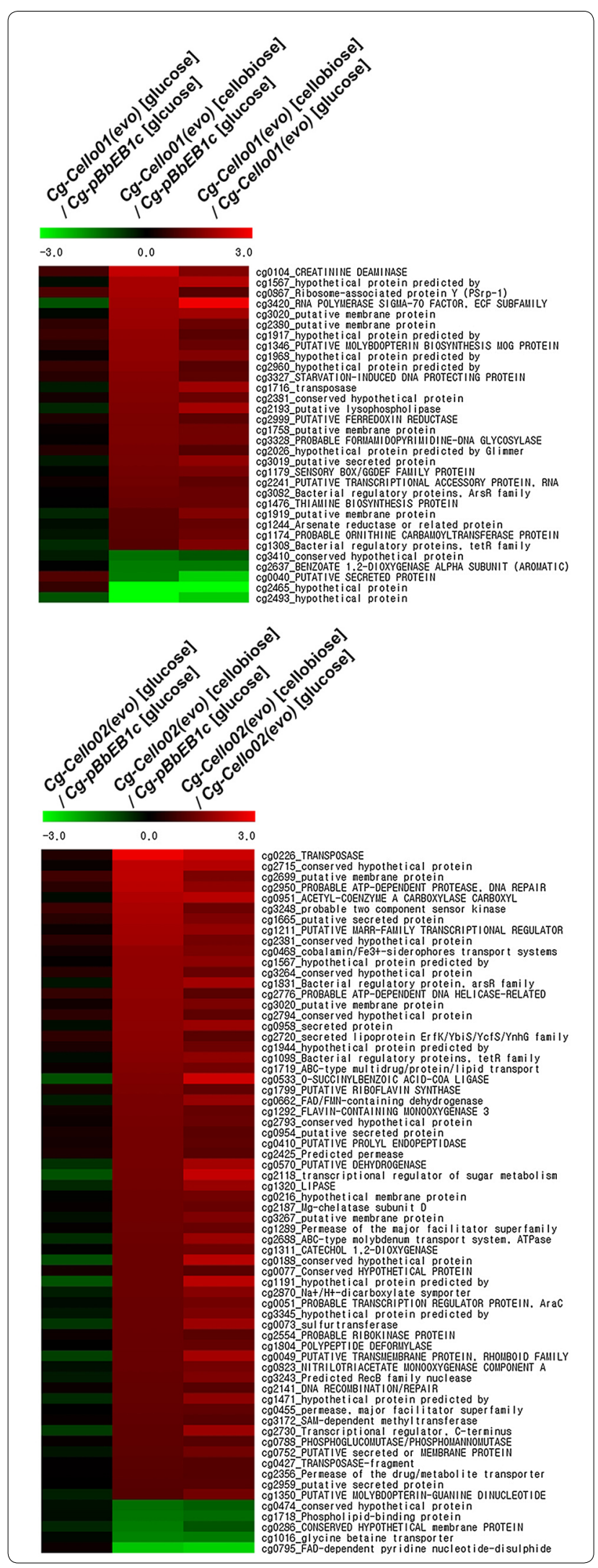


Table 3 Fatty acid profiles of the cellobiose-utilizing $C$. glutamicum strains and the wild-type

\begin{tabular}{lccc}
\hline $\begin{array}{l}\text { Fatty acid } \\
\text { composition (\%) }\end{array}$ & Cg-pBbEB1c & Cg-Cello01(evo) & Cg-Cello02(evo) \\
\hline$C_{10: 0}$ & 0.15 & 0.08 & 0.08 \\
$C_{12: 0}$ & 0.25 & - & - \\
$C_{12: 0} 30 H$ & - & 0.06 & 0.05 \\
$C_{14: 0}$ & 1.33 & 0.43 & 0.39 \\
$C_{16: 1}$ w9C & 0.84 & 0.51 & 0.46 \\
$C_{16: 0}$ & 41.04 & 35.89 & 33.28 \\
$C_{16: 0} 30 H$ & 0.31 & 0.69 & 0.34 \\
$C_{18: 1}$ w9C & 54.24 & 59.97 & 63.4 \\
$C_{18: 0}$ & 0.47 & 0.44 & 0.41 \\
$C_{18: 0}$ 10-methyl & 1.27 & 1.54 & 1.26 \\
$C_{19: 1}$ iso I & 0.09 & 0.33 & 0.33 \\
$C_{19: 1}$ w6C/unknown & - & 0.06 & - \\
fatty acids* & & & \\
\hline
\end{tabular}

The Cg-Cello01(evo) and Cg-Cello02(evo) strains were cultivated with $2 \%$ cellobiose as sole carbon source. The $\mathrm{Cg}$-pBbEB1c stain was cultivated with $2 \%$ glucose as sole carbon source. The fatty acid profiles of the $C g-p B b E B 1 c$ were almost identical to the profiles of the C. glutamicum wild-type [23]. Analysis of fatty acid and fatty acid methyl ester were followed by the standard protocol of Sherlock ${ }^{\circledR}$ Microbial Identification System (MIS) of MIcrobial IDentification Inc. (MIDI)

Data represents mean values of duplicated cultivations

* Equivalent chain lengths $(E C L)$ value $=18.846$

as its parental strain, $\mathrm{Cg}$-CelloO1(evo). Glucose derived from cellobiose was secreted into the medium while both $\mathrm{Cg}$-CelloO1(evo) and $\mathrm{Cg}$-CelloO3 strains consumed the cellobiose. In the case of $\mathrm{Cg}$-Cello04 strain, of which the parental strain is the $\mathrm{Cg}$-Cello02(evo) strain, the rates of cell growth and cellobiose consumption were slightly retarded but no glucose was detected as for the parental strain. The reason for slower cellobiose consumption remains unclear.

Instead of inverse engineering, we successfully constructed cellobiose-positive C. glutamicum chassis strain that utilize cellobiose as sole carbon source under the conditions of aerobic culture by expressing intracellular bG alone. Moreover, the strains expressing intracellular bG exhibited better cellobiose utilization than any other strain either secreting bG into the medium, or displaying bG on the cell surface [18] in terms of the cellobiose consumption rate under aerobic conditions. Therefore, we obtained cellobiose-positive C. glutamicum chassis strains to perform metabolic engineering with cellobiose as sole carbon source.

\section{Co-utilization of cellobiose and xylose in C. glutamicum through metabolic engineering}

Using the cellobiose-positive C. glutamicum chassis strains, we focused on co-utilization of xylose and cellobiose in C. glutamicum via direct cellobiose uptake and intracellular hydrolysis of cellobiose. As a result, $C g$ Cello03-XylO1 and Cg-Cello04-XylO1 strains were able to co-utilize cellobiose and xylose under aerobic conditions (Fig. 6). Compared to the xylose consumption by $\mathrm{Cg}$ $E c X y l A B$ in the presence of glucose, the xylose consumption rates of the engineered strains were improved in the presence of cellobiose, instead of glucose.

As shown in the culture of $C g$-EcXylAB strain with glucose and xylose, a biphasic growth of Cg-Cello03-Xyl01 strain was observed after $12 \mathrm{~h}$ when cellobiose was completely consumed. However, a biphasic growth behavior was not shown by Cg-Cello04-Xyl01 strain because the cellobiose was slowly consumed before $12 \mathrm{~h}$, and cellobiose and xylose were almost simultaneously utilized and depleted in the culture between 12 and $24 \mathrm{~h}$ (Fig. 6). Moreover, Cg-Cello04-Xyl01 strain reached higher optical density at first, compared to the Cg-CelloO3-Xyl01 strain. To further improve the engineered cellobiose- and xylose-utilizing strains, we introduced genes coding for sugar transporters (Gxf1 and Sut1) into Cg-Cello03-Xyl01 and $\mathrm{Cg}$-Cello04-XylO1 strains, respectively. But, the engineered strains with additional heterologous transporters did not show significant improvement of xylose utilization or co-utilization under the conditions of aerobic culture (Fig. 7).

Simultaneous consumption of cellobiose and xylose in engineered S. cerevisiae has resolved carbon catabolite repression and significantly increased ethanol productivity through co-fermentation [19]. Engineering of cellobiose-positive C. glutamicum also enabled the co-consumption of cellobiose and xylose (Figs. 6 and 7). However, compared to the cellobiose-utilizing S. cerevisiae, our cellobiose-positive C. glutamicum strains showed much faster cellobiose-consumption rates during aerobic culture. On the other hand, their xylose consumption rate was not much increased during co-fermentation. Expression of a pentose-specific transporter did not increase the rate, either. Therefore, we concluded that inefficient xylose utilization by $C$. glutamicum was another bottleneck for the co-fermentation of cellobiose and xylose although we did optimize the codon-usage of the corresponding Escherichia coli gene sequence [11]. Exploring an alternative xylose utilization pathway in $C$. glutamicum is necessary to provide faster xylose uptake $[13,20]$. This strategy could then be further applied for co-fermentation of xylose and cellobiose.

\section{Hydrolysis of Canadian biomass and efficient SSF by engineered $C$. glutamicum}

Taking advantage of the capability for cellobiose utilization by two engineered C. glutamicum strains $(\mathrm{Cg}$ CelloO3 and in Cg-Cello04), we applied the strains for 

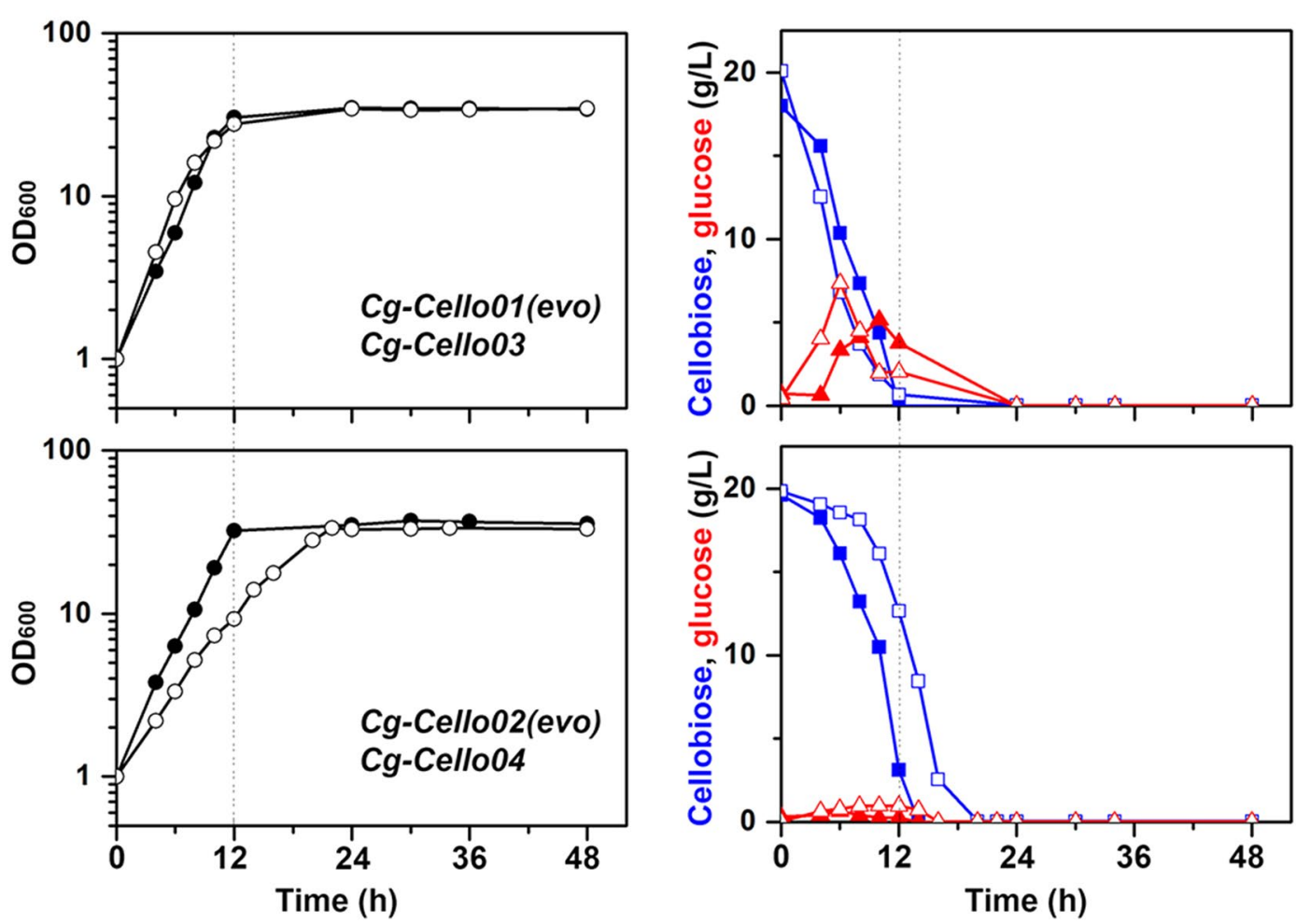

Fig. 5 A comparison of cell growth and cellobiose consumption of evolved C. glutamicum strains. The evolved C. glutamicum strains was compared with reconstructed cellobiose-positive chassis of C. glutamicum strains. By plasmid-curing and re-transformation of pBbEB1 c-bG plasmid, Cg-Cello03 (upper panels; open symbols) and Cg-Cello04 (lower panels; open symbols) strains as reconstructed cellobiose-positive chassis were obtained from Cg-Cello01 (evo) (upper panels; solid symbols) and Cg-Cello02(evo) (lower panels; solid symbols) strains, respectively. The strains were cultivated in CgXII medium with $2 \%(\mathrm{w} / \mathrm{v})$ cellobiose as sole carbon source after the pre-cultivation in BHIS medium. Growth (left panels; black circle), cellobiose (right panels; blue square), glucose (right panels; red triangle) were shown. Data represents mean values of at least three cultivations

efficient SSF of Canadian cellulosic hydrolysates. Before fermentation by C. glutamicum, we hydrolyzed either $1 \%$ (w/v) Avicel ${ }^{\circledR}$ PH-101, or $1 \%(\mathrm{w} / \mathrm{v})$ dissolving pulp (DP), with cellulase (Celluclast $1.5 \mathrm{~L}$ ) under the same culture conditions (except for the cell type). As a result, equal amounts of cellobiose and glucose were detected for both cellulosic substrates (Fig. 8; upper panels), and similar conversion yields of total sugar (16.7 \% and $16.15 \%)$ were obtained for Avicel and DP, respectively. Also, enzymatic hydrolysis terminated after $12 \mathrm{~h}$. There were not many differences between Avicel and DP as a cellulosic substrate for enzymatic hydrolysis with Celluclast $1.5 \mathrm{~L}$.

Based on the enzymatic hydrolysis, we investigated whether the engineered strains $(\mathrm{Cg}$-CelloO3 and in $\mathrm{Cg}$ Cello04) were able to utilize cellulosic substrates during SSF. Thus, we cultivated $1 \%(\mathrm{w} / \mathrm{v})$ cellulosic substrate of either Avicel or DP as sole carbon source for the evolved C. glutamicum strains with cellulase. No lag phase was shown by either culture (Fig. 8; lower panels). Compared to a control ( $C g-p B b E B 1 c$; the wild-type with empty vector), the CelloO3 and $\mathrm{Cg}$-CelloO4 strains showed faster growth, and reached nearly double growth at the end. When total amount of sugars was quantified independently, the total amount of sugars consumed in the $\mathrm{Cg}$-CelloO3 and $\mathrm{Cg}$ Cello04 cultures were higher than that of a control. Moreover, we measured cellobiose and glucose in the supernatant, with the result that no cellobiose and glucose were detected during the culture of CelloO3 and $\mathrm{Cg}$-CelloO4. Therefore, the engineered $\mathrm{Cg}$-CelloO3 and in $\mathrm{Cg}$-CelloO4 strains were able to grow by simultaneously utilizing cellobiose and glucose from cellulosic hydrolysates although low conversion yields of cellulosic substrates limit further cell growth during SSF. Along with improvements of the enzymatic hydrolysis, the simultaneous saccharification and co-fermentation (SSCF) of pretreated plant biomass (hexose and pentose) could be accomplished using engineered $C$. glutamicum strains $(C g$ Cello03-Xyl01 and Cg-Cello04-Xyl01).

\section{Conclusions}

Adaptive evolution of the microbial host to acquire desired environmental phenotypes was quite difficult unless growth-associated evolutions [25, 26]. In this study, integrated metabolic engineering and adaptive evolution allowed us to develop a cellobiose- and 

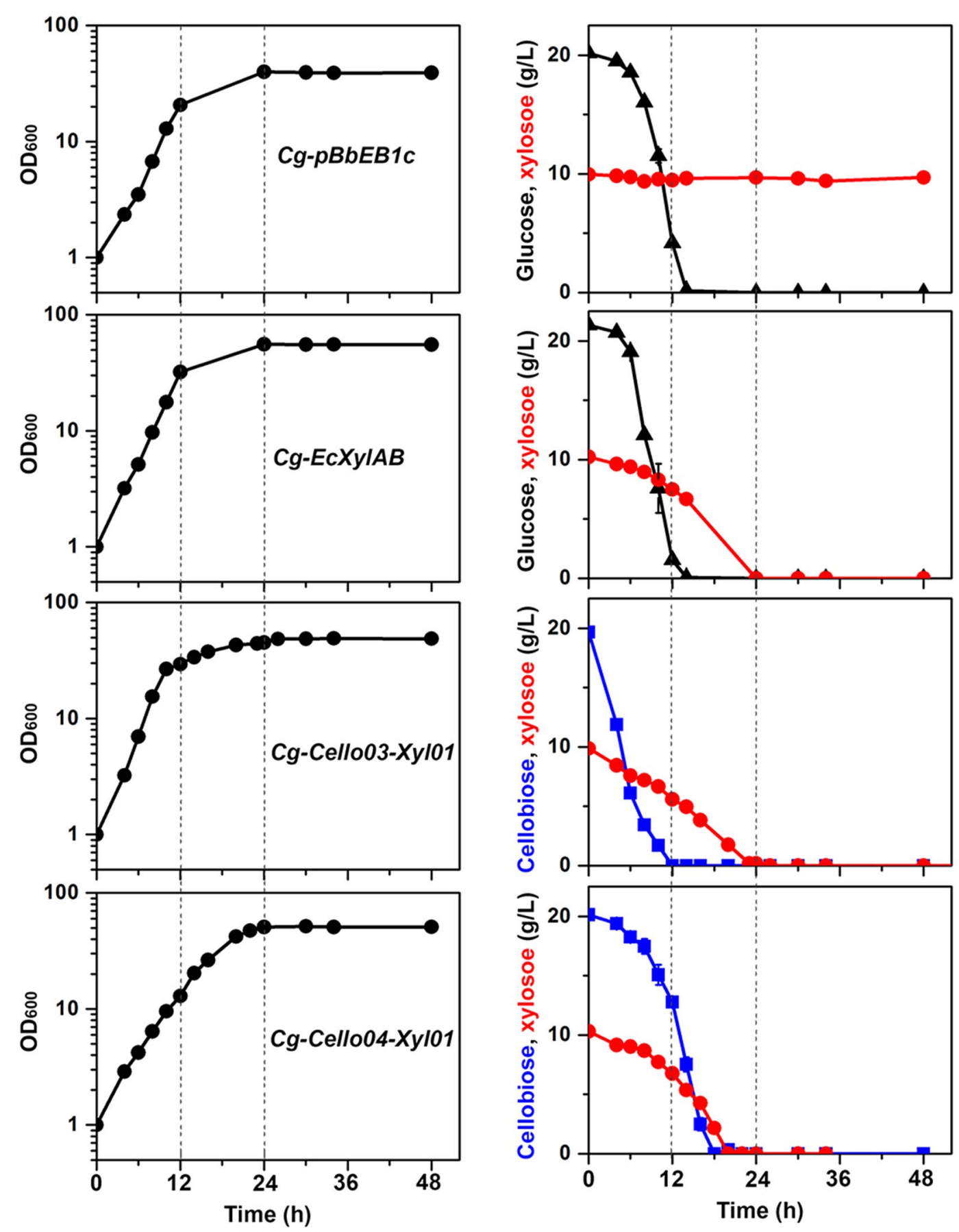

Fig. 6 Co-consumption of cellobiose and xylose of engineered cellobiose-positive chassis of C. glutamicum strains. Two different cellobiose-negative strains (Cg-pBbEB1C and Cg-ECXYlAB) and two different cellobiose-positive strains (Cg-Cello03-Xyl01 and Cg-Cello04-Xyl01) co-expressing XylA and XylB were tested. The cellobiose-negative strains were cultivated in CgXII medium with a mixture of $2 \%(\mathrm{w} / \mathrm{v})$ glucose and $1 \%(\mathrm{w} / \mathrm{v}) \mathrm{xylose}$. On the other hand, the cellobiose-positive strains were cultivated in CgXII medium with a mixture of $2 \%(\mathrm{w} / \mathrm{v})$ cellobiose and $1 \%$ (w/v) xylose. Growth (left panels; black circle), cellobiose (right panels; blue square), glucose (right panels; black triangle), xylose (right panels; red circle) were shown. Data represents mean values of at least three cultivations and error bars represent standard deviations

xylose-negative C. glutamicum strain that co-utilize cellobiose and xylose using. For further studies, we envision development of recombinant C. glutamicum strains based on the chassis strain, for efficient lignocellulosicbiomass conversion to create valuable products such as L-glutamate or L-lysine. 

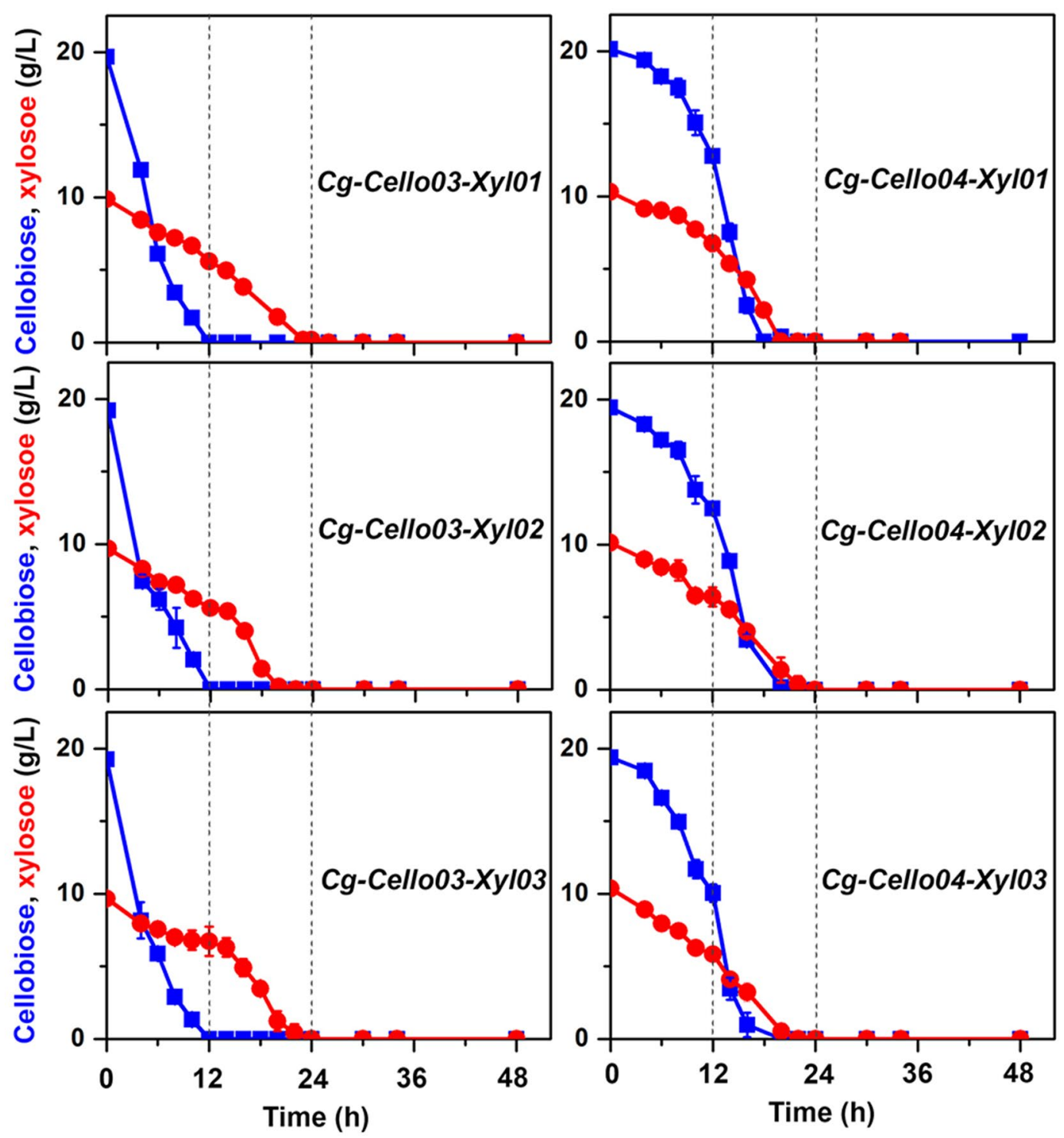

Fig. 7 Additional sugar transporters for co-consumption of cellobiose and xylose. Two different cellobiose-positive strains [Cg-Cello03-Xyl01 (top left panel) and Cg-Cello04-Xyl01 (top right panel)] co-expressing XylA and XylB were further engineered with additional sugar transporters such as a Gxf1 (Candia intermedia) [29] and Sut1 (Pichia stipites) [30], yielding Cg-Cello03-Xyl02 and Cg-Cello03-Xyl03 (left panels) and Cg-Cello04-Xyl02 and CgCello04-Xyl03 (right panels), respectively. The cellobiose-positive and xylose-positive strains were cultivated in CgXII medium with a mixture of $2 \%$ $(\mathrm{w} / \mathrm{v})$ cellobiose and $1 \%(\mathrm{w} / \mathrm{v})$ xylose. Cellobiose (blue square), xylose (red circle) were shown. Data represents mean values of at least three cultivations and error bars represent standard deviations

\section{Methods}

\section{Bacterial strains, plasmids and culture conditions}

All bacterial strains and plasmids used or constructed in this work are listed in Table 1. E. coli strains were grown in LB medium (containing per liter: $10 \mathrm{~g}$ tryptone, $5 \mathrm{~g}$ yeast extract, and $5 \mathrm{~g} \mathrm{NaCl}$ ) at $37{ }^{\circ} \mathrm{C}$ and $200 \mathrm{rpm}$. $C$. glutamicum ATCC 13032 and its derivatives were cultivated in BHIS medium (containing per liter: $37 \mathrm{~g}$ brain heart infusion, $91 \mathrm{~g}$ sorbitol) [27] at $30{ }^{\circ} \mathrm{C}$ and $200 \mathrm{rpm}$ overnight and then incubated aerobically in CgXII medium (50 in $250 \mathrm{~mL}$ baffled Erlenmeyer flasks) [27] containing $2 \%(\mathrm{w} / \mathrm{v})$ cellobiose or a mixture of $2 \%(\mathrm{w} / \mathrm{v})$ cellobiose and $1 \%(\mathrm{w} / \mathrm{v})$ xylose supplemented with $25 \mu \mathrm{g} / \mathrm{mL}$ chloramphenicol at $30{ }^{\circ} \mathrm{C}$ on a rotary shaker at $200 \mathrm{rpm}$. All chemicals used in this study were purchased from Sigma-Aldrich (St. Louis, Mo). $0.5 \mathrm{mM}$ isopropyl- $\beta$-D-thiogalactopyranoside (IPTG) was added for induction. 

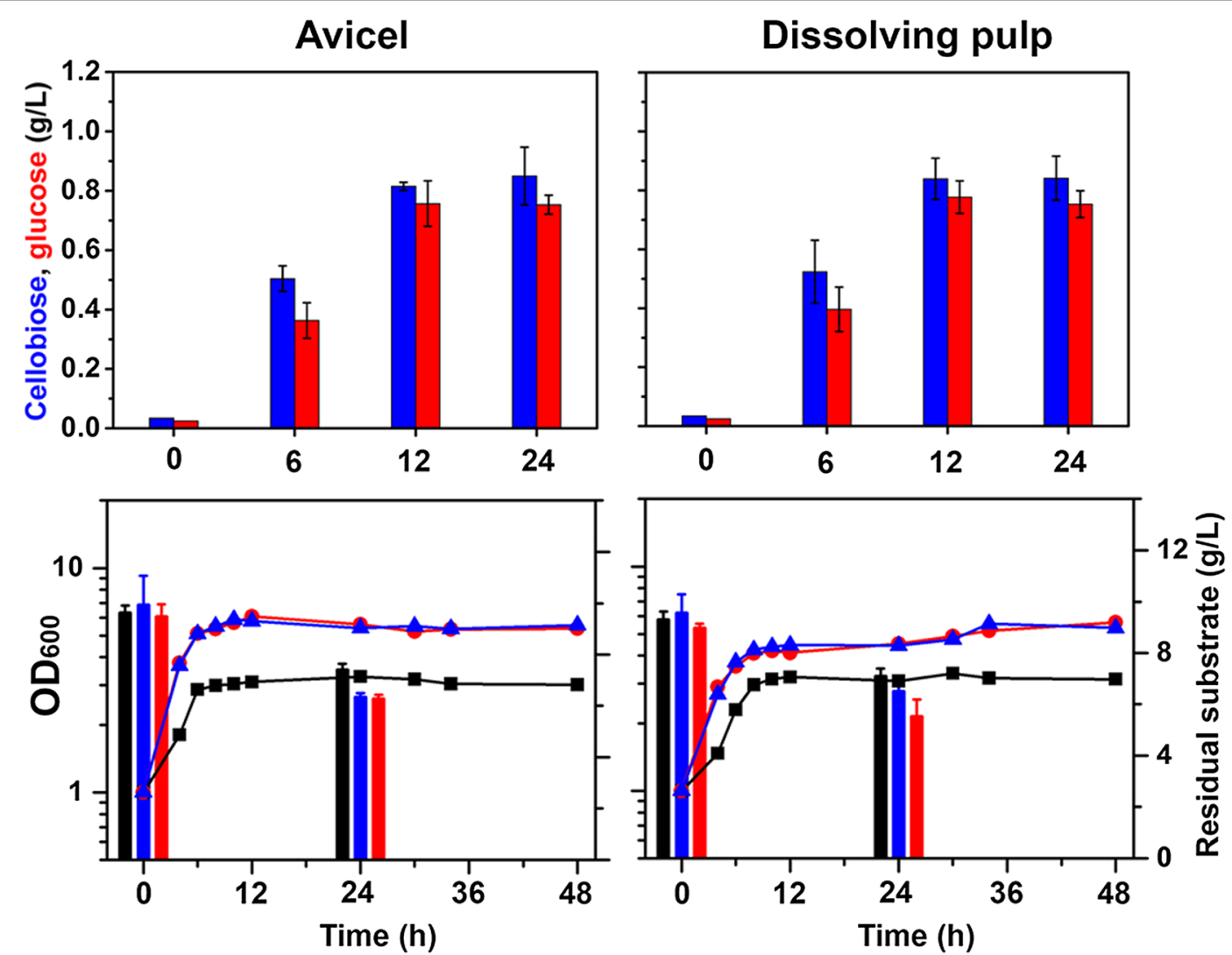

Fig. 8 Profiles of conversion of Avice ${ }^{\circledR}$ PH-101 or DP by C. glutamicum strains. Celluclast 1.5 L (Sigma; Cat C2730) [75 filter paper unit (FPU)/gglucan] was used as the cellulolytic enzymes for saccharification of Avice ${ }^{\circledR} \mathrm{PH}-101$ (left panels) or DP (right panels). For cellulolytic hydrolysis (upper panels), Avicel $(1 \%[\mathrm{w} / \mathrm{V}])$ or DP $(1 \%[\mathrm{w} / \mathrm{v}])$ were hydrolyzed at $30^{\circ} \mathrm{C}$ and cellobiose (blue bar) and glucose (red bar) were measured. For SSF (lower panels), Cg-pBbEB1c (black square), Cg-Cello03 (blue triangle) and Cg-Cello04 (red circle) were cultivated with either Avicel (1 \% [w/v]) or DP (1 \% [w/v]) as sole carbon source in the presence of Celluclast $1.5 \mathrm{~L}$ and optical densities at $600 \mathrm{~nm}$ were measured after the sedimentation of the residual substrate (lower panels; lines and symbols with left $Y$-axis). For the measurement of the residual substrate $(\mathrm{g} / \mathrm{L}$ ), each residual substrate was measured at 0 and $24 \mathrm{~h}$ from the SSF cultures (lower panels; bars with right Y-axis). During SSF, no cellobiose and glucose were detected in the supernatant from the cultures. Data represents mean values of at least three cultivations and error bars represent standard deviations

\section{Construction of plasmids and recombinant C. glutamicum strains}

The $c d t-1$ (NCU00801) and gh1-1 (NCU00130) genes from Neurospora crassa [19] encoding for a cellodextrin transporter and a bG, respectively, were chosen for constructing a synthetic pathway in C. glutamicum. Each target gene was synthesized (Genscript, USA) with codon-optimization (Gene Designer 2.0 software [28]) for C. glutamicum and was assembled using a standard BglBrick cloning method, where the target gene is inserted at the EcoRI and XhoI sites of the CoryneBrick plasmid pBbEB1c [11]. Thus, the synthesized gh1-1 gene was inserted, resulting pBbEB1c-bG. Subsequently, the synthesized $c d t$ - 1 gene was placed in front of the $g h 1-1$ gene, resulting pBbEB1c-CT-bG. For utilization of xylose, the codon-optimized $x y l A$ and $x y l B$ genes from $E$. coli [11] were, subsequently, inserted to pBbEB1c-bG, resulting
pBbEB1c-bG-XIK. In addition, the gxf1 (Candia intermedia) [29] and sut1 (Pichia stipites) [30] gene encoding for sugar-transporter were codon-optimized and inserted to pBbEB1c-bG-XIK, yielding pBbEB1c-bG-XIK-XTg and pBbEB1c-bG-XIK-XTs, respectively. Cloned DNA fragments were correctly verified by DNA sequencing.

The resulting plasmids were introduced into $C$. glutamicum by electroporation, and strain validation was performed by colony PCR [27]. The resulting strains are listed in Table 1.

\section{Adaptive Evolution of recombinant $C$. glutamicum strains}

$\mathrm{Cg}$-CelloO1 and $\mathrm{Cg}$-CelloO2 strains were cultivated in CgXII minimal medium containing $2 \%(\mathrm{w} / \mathrm{v})$ cellobiose as sole carbon source. After the maximal cell growth of Cg-CelloO1 and Cg-CelloO2 were observed in 16 and 11 days, respectively, the cells were transferred to the 
fresh CgXII minimal medium containing $2 \%(\mathrm{w} / \mathrm{v})$ cellobiose, starting $\mathrm{OD}_{600}$ of 1 (Fig. 2a). Subsequently, the cells were transferred to the same fresh medium after every $48 \mathrm{~h}$. Each culture of the cell was analyzed using HPLC to investigate the changes of the profiles of cellobiose utilization. The cell transfers were conducted until the rates of growth and cellobiose consumption were not changed, yielding $\mathrm{Cg}$-Cello01(evo) and $\mathrm{Cg}$-CelloO2(evo) strains. Cg-Cello01(evo) and Cg-CelloO2(evo) strains were further analyzed using DNA microarray. Plasmids from $\mathrm{Cg}$-CelloO1(evo) and $\mathrm{Cg}$-Cello02(evo) strains were isolated and their DNA sequences were identified using partial overlapping primer-walking. In addition, plasmid-free strains were obtained by curing of plasmids in C. glutamicum as follows: after the electroporation of Cg-Cello01(evo) and Cg-CelloO2(evo) strains, nonselective BHIS medium was inoculated at $30{ }^{\circ} \mathrm{C}$. Each single colony was streaked onto BHIS agar plates either with or without chloramphenicol $(25 \mu \mathrm{g} / \mathrm{mL})$, yielding plasmidfree $\left(\mathrm{Cm}^{\mathrm{s}}\right) \mathrm{Cg}$-Evo1 and $C g$-Evo 2 strains, respectively. For co-utilization of cellobiose and xylose, the xylA (encoding for xylose isomerase) and $x y l B$ (encoding for xylulose kinase) genes was introduced into cellobiose-utilizing $\mathrm{Cg}$-CelloO3 and $\mathrm{Cg}$-CelloO4 strains, yielding $\mathrm{Cg}$-CelloO3Xyl01 and Cg-Cello04-Xyl01 strains.

\section{HPLC analysis for glucose, xylose, and cellobiose quantification}

For the measurement of the concentrations of glucose, xylose and cellobiose, culture supernatant was passed through a syringe filter (pore size of $0.2 \mu \mathrm{m}$ ) after centrifugation at $10,000 \mathrm{~g}$ for $10 \mathrm{~min}$. The concentrations of glucose and xylose were determined by high-performance liquid chromatography (HPLC system Agilent 1260, Waldbronn, Germany) equipped with a refractive index detector (RID) and an Aminex HPX-87 H Ion Exclusion Column (300 mm by $7.8 \mathrm{~mm}$, Bio-Rad, Hercules, CA, USA) under the following conditions: sample volume of $20 \mu \mathrm{L}$, mobile phase of $5 \mathrm{mM} \mathrm{H}_{2} \mathrm{SO}_{4}$, flow rate of $0.6 \mathrm{~mL} /$ min, and column temperature of $65^{\circ} \mathrm{C}$.

\section{Enzymatic measurement of $\beta$-glucosidase and glucokinase activity}

Recombinant strains were cultivated in CgXII medium containing $2 \%(\mathrm{w} / \mathrm{v})$ cellobiose but $2 \%(\mathrm{w} / \mathrm{v})$ glucose was used for the control $(C g-p B b E B 1 c)$. After incubation at $30{ }^{\circ} \mathrm{C}$ for $24 \mathrm{~h}$, bG activities in the cell-free extracts or in the culture supernatants, respectively, were quantitatively measured in a $1 \mathrm{~mL}$ mixture containing $590 \mu \mathrm{L}$ $500 \mathrm{mM}$ potassium phosphate buffer $(\mathrm{pH} 7.0), 10 \mu \mathrm{L}$ $500 \mathrm{mM} \mathrm{MgCl}, 200 \mu \mathrm{L}$ sample, $200 \mu \mathrm{L} p$-nitrophenyl- $\beta$ d-glucopyranoside ( $p$ NPG) as a substrate at $410 \mathrm{~nm}$ [16] $\left(\mathrm{U} ; \mu \mathrm{mol}\right.$ of $p$ NPG reduced $\mathrm{min}^{-1}$ ). For the determination of Glk activity (U/L) [31], the Glk activity in cell-free extracts was determined at $25{ }^{\circ} \mathrm{C}$ by measuring of the formation of NADPH at $340 \mathrm{~nm}$ in a coupled reaction containing $100 \mathrm{mM}$ potassium phosphate buffer ( $\mathrm{pH}$ 7.0), $20 \mathrm{mM}$ glucose, $2 \mathrm{mM}$ ATP, $25 \mathrm{mM} \mathrm{MgCl}$, 2 mM NADP and $2 \mathrm{U}$ glucose-6-phosphate dehydrogenase (U; $\mu \mathrm{mol}$ of NADP reduced $\mathrm{min}^{-1}$ ).

\section{NGS-based genomic DNA sequencing analysis}

Genomic DNAs of Cg-Cello01(evo) and Cg-CelloO2(evo) were isolated from a single colony's culture and purified using Wizard Genomic DNA purification kit (Promega, Cat.No. A1125). The genomes of Cg-CelloO1(evo) and $\mathrm{Cg}$-Cello02(evo) strains were sequenced using the Illumina Miseq 300 bp paired-end system (Illumina, Inc, San Diego, CA, USA) at ChunLab, Inc. (Seoul, South Korea). We obtained 5,753,368 reads of the genome to reach a 428.63-fold depth of coverage on Cg-CelloO1(evo) and $\mathrm{Cg}$-Cello02(evo). The re-sequencing data were annotated by RNAmmer 1.2, tRNA scan-SE 1.21, Rapid Annotation using Subsystem Technology (RAST), Pipeline, and CLgenomics program (ChunLab, Inc). The detail procedures were described in the previous study [32].

\section{Transcriptomic analysis}

Total RNA from Cg-Cello01(evo) and Cg-CelloO2(evo) were sampled in the exponential phase. For Transcriptome analysis, extraction of total RNA and preparation of cDNA was followed by previous methods [33]. The cDNA probes were cleaned up using Microcon YM-30 column (Millipore, Bedford, MA) and then followed by Cy5-dye (Amersham Pharmacia, Uppsala, Sweden). The Cy5-labelled cDNA probes were cleaned up using the QIAquick PCR Purification Kit (Qiagen). Dried Cy5-labelled cDNA probes were re-suspended in hybridization buffer. Hybridization to a microarray slide (Corynebacterium_glutamicum $3 \times 20 \mathrm{~K}$ ) (Mycroarray.com, Ann Arbor, MI), staining, and scanning of the probe array were performed according to the manufacturer's instructions. Hybridization image on the slide was scanned by Axon 4000B (Axon Instrument, Union City, CA). The analysis of the microarray data was performed using GenePix Pro 6.0 (Axon Instruments). The averages of the normalized ratios were calculated by dividing the average normalized signal channel intensity by the average normalized control channel intensity. All measurements were performed on duplicated technical replicates. Two independent samples were analyzed; their measurements were averaged for further analysis. The microarray data were deposited at the NCBI Gene Expression Omnibus, GEO under accession no. GSE65076 and at http://www.ncbi.nlm.nih.gov/geo/ query/acc.cgi?acc $=$ GSE65076. 


\section{Fatty acids and lipid analysis}

Fatty acid methyl esters were prepared as described previously [23, 34], and identified by gas chromatography with model 5898A microbial identification system (Microbial ID). Trimethylsilylated derivatives of fatty acids and methyl esters were analyzed by hightemperature gas chromatography on an HP 5790A gas chromatograph (Hewlett Packard), equipped with a flame-ionization detector on a $12 \mathrm{~m}$ high throughput screening (HTS) column with hydrogen gas as the carrier. Derivatives were identified by comparing their retention times to those of standards and by gas chromatography mass spectrometry analysis on a KRATOS MS50 spectrometer (ion source temperature set to $200{ }^{\circ} \mathrm{C}$ and ionization energy to $70 \mathrm{eV})$, respectively. For the analysis, colonies of $\mathrm{Cg}$-Cello01(evo) and $\mathrm{Cg}$-Cello02(evo) strains were obtained on CgXII agar plate containing $2 \%$ cellobiose and colonies of $C g-p B b E B 1 c$ stain was obtained on CgXII agar plate containing $2 \%$ glucose as.

\section{Cellulolytic hydrolysis of Avicel ${ }^{\circledR} \mathrm{PH}-101$ and Canadian biomass and SSF by C. glutamicum}

Avicel $^{\circledR}$ PH-101 (Sigma), microcrystalline cellulose and dissolving pulp (DP, pure cellulosic substrate less than $0.5 \%$ lignin, less than 3 \% xylan) [35] from Canadian Ponderosa Pine were used as substrate for cellulolytic hydrolysis and SSF by the cellobiose-utilizing C. glutamicum strains. Each cellulolytic hydrolysis and SSF was carried out in CgXII medium (pH 7.0) with $1 \%$ (w/v) Avicel ${ }^{\circledR}$ $\mathrm{PH}-101$ or $1 \%(\mathrm{w} / \mathrm{v})$ dissolvping pulp at $30{ }^{\circ} \mathrm{C}$ and 200 rpm. Celluclast 1.5 L (Sigma; Cat C2730) [75 filter paper unit (FPU)/g-glucan] was used as the cellulolytic enzymes for saccharification of Avicel ${ }^{\circledR}$ PH-101 or DP. Cellulase actitivity of the Celluclast $1.5 \mathrm{~L}$ was determined by the standard filter paper assay with the 3,5-dinitrosalicylic acid (DNS) method [36]. One unit of cellulose activity is defined as the amount of enzyme required to release $1 \mu \mathrm{mol}$ of reducing sugar per mint at $\mathrm{pH} 4.8$ and $50{ }^{\circ} \mathrm{C}$. The enzyme activity of Celluclast $1.5 \mathrm{~L}$ was measured to be $28 \mathrm{FPU} / \mathrm{mL}$. A colorimetric method based on the phenol-sulfuric acid reaction was used to determine the amount of residual substrate during SSF by quantifying total sugars [37].

\section{Availability of supporting data}

The data set supporting the results of this article is available at NCBI GEO repository, [GSE65076 and http://www. ncbi.nlm.nih.gov/geo/query/acc.cgi?acc=GSE65076].

\section{Additional file}

Additional file 1. Additional data and analysis for the evolved $C$. glutamicum strains.

\section{Abbreviations}

bG: $\beta$-glucosidase; SSF: simultaneous saccharification and fermentation; HPLC: high-performance liquid chromatography; RID: refractive index detector; NGS: next-generation sequencing; GEO: gene expression omnibus; Glk: glucokinase; DP: dissolving pulp; SSCF: simultaneous saccharification and co-fermentation.

\section{Authors' contributions}

$J$ performed and analyzed all experiments and helped in drafted the manuscript. JNS and YU assisted in analysis and helped in drafting the manuscript. HMW analyzed all experiments and coordinated the study and drafted the manuscript. All authors read and approved the final manuscript.

\section{Author details}

${ }^{1}$ Clean Energy Research Center, Korea Institute of Science and Technology (KIST), Hwarangro 14-gil 5, Seongbuk-gu, Seoul 02792, Republic of Korea. 2 Department of Wood Science, University of British Columbia, Vancouver, BC V6T 1Z4, Canada. ${ }^{3}$ Department of Clean Energy and Chemical Engineering, Korea University of Science and Technology (UST), 217 Gajeong-ro, Yuseong-gu, Daejeon 34113, Republic of Korea. ${ }^{4}$ Green School (Graduate School of Energy and Environment), Korea University, 145 Anam-ro, Seongbuk-gu, Seoul 02841, Republic of Korea.

\section{Acknowledgements}

Authors appreciate to Dr. Changsoo Kim at UBC-KIST on-site laboratory for valuable comments and also thank to Dr. Jinguang Hu at UBC for sending DP (Canadian biomass). This work was financially supported from the R\&D Convergence Program of NST (National Research Council of Science and Technology) of Republic of Korea and KIST (Korea Institute of Science and Technology) (2E25402) and Korea CCS R\&D Center (KCRC) (no. 2014M1A8A1049277) and the National Research Foundation of Korea grant-funded by the Korean Government (Ministry of Science, ICT and Future Planning) (2015, UniversityInstitute Cooperation program).

\section{Competing interests}

The authors declare that they have no competing interests.

Received: 9 January 2016 Accepted: 11 January 2016

Published online: 22 January 2016

\section{References}

1. Steen EJ, Kang Y, Bokinsky G, Hu Z, Schirmer A, McClure A, Del Cardayre SB, Keasling JD. Microbial production of fatty-acid-derived fuels and chemicals from plant biomass. Nature. 2010;463:559-62.

2. Peralta-Yahya PP, Zhang F, Del Cardayre SB, Keasling JD. Microbial engineering for the production of advanced biofuels. Nature. 2012;488:320-8.

3. Wang HH, Isaacs FJ, Carr PA, Sun ZZ, Xu G, Forest CR, Church GM. Programming cells by multiplex genome engineering and accelerated evolution. Nature. 2009;460:894-8.

4. Sander JD, Joung JK. CRISPR-Cas systems for editing, regulating and targeting genomes. Nat Biotechnol. 2014;32:347-55.

5. Carroll A, Somerville C. Cellulosic biofuels. Annu Rev Plant Biol. 2009;60:165-82.

6. Ikeda M, Nakagawa S. The Corynebacterium glutamicum genome: features and impacts on biotechnological processes. Appl Microbiol Biotechnol. 2003:62:99-109.

7. Blombach B, Seibold GM. Carbohydrate metabolism in Corynebacterium glutamicum and applications for the metabolic engineering of L-lysine production strains. Appl Microbiol Biotechnol. 2010;86:1313-22.

8. Becker J, Wittmann C. Bio-based production of chemicals, materials and fuels-Corynebacterium glutamicum as versatile cell factory. Curr Opin Biotechnol. 2012;23:631-40.

9. Wieschalka S, Blombach B, Bott M, Eikmanns BJ. Bio-based production of organic acids with Corynebacterium glutamicum. Microb Biotechnol. 2013:6:87-102.

10. Woo HM, Park JB. Recent progress in development of synthetic biology platforms and metabolic engineering of Corynebacterium glutamicum. J Biotechnol. 2014;180:43-51. 
11. Kang MK, Lee J, Um Y, Lee TS, Bott M, Park SJ, Woo HM. Synthetic biology platform of CoryneBrick vectors for gene expression in Corynebacterium glutamicum and its application to xylose utilization. Appl Microbiol Biotechnol. 2014;98:5991-6002.

12. Kawaguchi $H$, Vertès $A A$, Okino $S$, Inui $M$, Yukawa $H$. Engineering of a xylose metabolic pathway in Corynebacterium glutamicum. Appl Environ Microbiol. 2006;72:3418-28.

13. Radek A, Krumbach K, Gatgens J, Wendisch VF, Wiechert W, Bott M, Noack $\mathrm{S}$, Marienhagen J. Engineering of Corynebacterium glutamicum for minimized carbon loss during utilization of d-xylose containing substrates. J Biotechnol. 2014;192 Pt A:156-60.

14. Kotrba P, Inui M, Yukawa H. The ptsI gene encoding enzyme I of the phosphotransferase system of Corynebacterium glutamicum. Biochem Biophys Res Commun. 2001;289:1307-13.

15. Kotrba $P$, Inui M, Yukawa H. A single V317A or V317 M substitution in Enzyme II of a newly identified beta-glucoside phosphotransferase and utilization system of Corynebacterium glutamicum R extends its specificity towards cellobiose. Microbiology. 2003;149:1569-80.

16. Sasaki M, Jojima T, Inui M, Yukawa H. Simultaneous utilization of D-cellobiose, D-glucose, and D-xylose by recombinant Corynebacterium glutamicum under oxygen-deprived conditions. Appl Microbiol Biotechnol. 2008;81:691-9.

17. Kalinowski J, Bathe B, Bartels D, Bischoff N, Bott M, Burkovski A, Dusch N, Eggeling L, Eikmanns BJ, Gaigalat L, et al. The complete Corynebacterium glutamicum ATCC 13032 genome sequence and its impact on the production of L-aspartate-derived amino acids and vitamins. J Biotechnol. 2003;104:5-25.

18. Adachi N, Takahashi C, Ono-Murota N, Yamaguchi R, Tanaka T, Kondo A. Direct L-lysine production from cellobiose by Corynebacterium glutamicum displaying beta-glucosidase on its cell surface. Appl Microbiol Biotechnol. 2013;97:7165-72.

19. Ha SJ, Galazka JM, Kim SR, Choi JH, Yang X, Seo JH, Glass NL, Cate JH, Jin YS. Engineered Saccharomyces cerevisiae capable of simultaneous cellobiose and xylose fermentation. Proc Natl Acad Sci U S A. 2011;108:504-9.

20. Meiswinkel TM, Gopinath V, Lindner SN, Nampoothiri KM, Wendisch VF. Accelerated pentose utilization by Corynebacterium glutamicum for accelerated production of lysine, glutamate, ornithine and putrescine. Microb Biotechnol. 2013;6:131-40.

21. Tanaka Y, Okai N, Teramoto H, Inui M, Yukawa H. Regulation of the expression of phosphoenolpyruvate: carbohydrate phosphotransferase system (PTS) genes in Corynebacterium glutamicum R. Microbiology. 2008;154:264-74.

22. Kohl TA, Baumbach J, Jungwirth B, Puhler A, Tauch A. The GlxR regulon of the amino acid producer Corynebacterium glutamicum: in silico and in vitro detection of DNA binding sites of a global transcription regulator. J Biotechnol. 2008;135:340-50.

23. Radmacher E, Alderwick LJ, Besra GS, Brown AK, Gibson KJ, Sahm H, Eggeling L. Two functional FAS-I type fatty acid synthases in Corynebacterium glutamicum. Microbiology. 2005;151:2421-7.

24. Hetzler S, Steinbuchel A. Establishment of cellobiose utilization for lipid production in Rhodococcus opacus PD630. Appl Environ Microbiol. 2013;79:3122-5.

25. Caspeta L, Chen Y, Ghiaci P, Feizi A, Buskov S, Hallstrom BM, Petranovic D, Nielsen J. Altered sterol composition renders yeast thermotolerant. Science. 2014;346:75-8.

26. Lam FH, Ghaderi A, Fink GR, Stephanopoulos G. Engineering alcohol tolerance in yeast. Science. 2014;346:71-5.
27. Eggeling L, Bott M. Handbook of Corynebacterium glutamicum. CRC press; 2010.

28. Welch M, Govindarajan S, Ness JE, Villalobos A, Gurney A, Minshull J, Gustafsson C. Design parameters to control synthetic gene expression in Escherichia coli. PLoS One. 2009;4:e7002.

29. Runquist D, Fonseca C, Radstrom P, Spencer-Martins I, Hahn-Hagerdal B. Expression of the Gxf1 transporter from Candida intermedia improves fermentation performance in recombinant xylose-utilizing Saccharomyces cerevisiae. Appl Microbiol Biotechnol. 2009;82:123-30.

30. Katahira S, Ito M, Takema H, Fujita Y, Tanino T, Tanaka T, Fukuda H, Kondo A. Improvement of ethanol productivity during xylose and glucose cofermentation by xylose-assimilating S. cerevisiae via expression of glucose transporter Sut1. Enzyme Microb Technol. 2008;43:115-9.

31. Park SY, Kim HK, Yoo SK, Oh TK, Lee JK. Characterization of glk, a gene coding for glucose kinase of Corynebacterium glutamicum. FEMS Microbiol Lett. 2000;188:209-15.

32. Jeon YS, Park SC, Lim J, Chun J, Kim BS. Improved pipeline for reducing erroneous identification by 165 rRNA sequences using the Illumina MiSeq platform. J Microbiol. 2015;53:60-9.

33. Park H-S, Um Y, Sim SJ, Lee SY, Woo HM. Transcriptomic analysis of Corynebacterium glutamicum in the response to the toxicity of furfural present in lignocellulosic hydrolysates. Proc Biochem. 2015;50:347-56.

34. Klatte S, Kroppenstedt RM, Rainey FA. Rhodococcus opacus sp.nov., An unusual nutritionally versatile Rhodococcus-species. Syst Appl Microbiol. 1994;17:355-60.

35. Hu JG, Arantes V, Pribowo A, Saddler JN. The synergistic action of accessory enzymes enhances the hydrolytic potential of a "cellulase mixture" but is highly substrate specific. Biotechnol Biofuels. 2013;6:112.

36. Ghose TK. Measurement of Cellulase Activities. Pure Appl Chem 1987;59:257-68.

37. DuBois M, Gilles KA, Hamilton JK, Rebers PA, Smith F. Colorimetric method for determination of sugars and related substances. Anal Chem. 1956;28:350-6.

38. Hanahan D. Studies on transformation of Escherichia coli with plasmids. J Mol Biol. 1983; 166:557-80.

39. Lee J, Sim SJ, Bott M, Um Y, Oh MK, Woo HM. Succinate production from CO2-grown microalgal biomass as carbon source using engineered Corynebacterium glutamicum through consolidated bioprocessing. Sci Rep. 2014;4:5819.

\section{Submit your next manuscript to BioMed Central and we will help you at every step:}

- We accept pre-submission inquiries

- Our selector tool helps you to find the most relevant journal

- We provide round the clock customer support

- Convenient online submission

- Thorough peer review

- Inclusion in PubMed and all major indexing services

- Maximum visibility for your research

Submit your manuscript at www.biomedcentral.com/submit

C BioMed Central 